\title{
Medication Reconciliation in Oncological Patients: A Randomized Clinical Trial
}

\author{
Triana González-Carrascosa Vega, Pharmacist; Jesús Francisco Sierra-Sánchez, PharmD; \\ María José Martínez-Bautista, BCOP Pharmacist; Fátima García-Martín, Pharmacist; \\ Francisco Suárez-Carrascosa, Pharmacist; and Jose Manuel Baena-Cañada, PhD
}

\begin{abstract}
BACKGROUND: Medication reconciliation is considered to be an important strategy for increasing the safety of medication use. However, few studies have been carried out showing the effect of a medication reconciliation program on the incidence of reconciliation errors (REs) in oncological patients treated in the outpatient setting.

OBJECTIVE: To measure the effect of a medication reconciliation program on the incidence of reconciliation error that reached the patient (RERP) in cancer patients receiving chemotherapy as outpatients.

METHODS: A randomized, prospective, controlled study was carried out to identify the proportion of patients with at least 1 RERP. Medication reconciliation (intervention group) was compared with standard practice (control group) in patients starting new chemotherapy and who were receiving at least 1 home medication before the start of chemotherapy. A prespecified analysis of factors capable of influencing the occurrence of RE in oncological patients was also carried out.

RESULTS: A total of 147 patients were included (76 in the intervention group and 71 controls) in this study. There were $3(4 \%)$ patients with RERP (primary endpoint) in the intervention group and $21(30 \%)$ patients in the control group (relative risk $[\mathrm{RR}]=0.13,95 \% \mathrm{Cl}=0.04-0.43 ; P=0.0009$ ). The prespecified analysis of the effects of the Eastern Cooperative Oncology Group performance status (ECOG), Charlson Comorbidity Index score, and degree of poly-medication upon the number of patients with RE showed the Charlson Comorbidity Index to be unrelated to RE occurrence. However, the risk of $\mathrm{RE}$ was greater in patients with $\mathrm{ECOG} \geq 2(\mathrm{RR}=2.18,95 \% \mathrm{Cl}=$ 1.4-3.4; $P=0.018)$ and among patients with major poly-medication (RR=2.49, 95\% Cl=1.52-4.09; $P<0.001)$.

CONCLUSIONS: Medication reconciliation results in a marked decrease in RERP in cancer patients. The factors that may influence RE occurrence in oncological patients have not been fully established, although parameters such as the degree of poly-medication and performance status may play a role.
\end{abstract}

J Manag Care Spec Pharm. 2016;22(6):734-40

Copyright @ 2016, Academy of Managed Care Pharmacy. All rights reserved.

\section{What is already known about this subject}

Medication reconciliation has been shown to reduce medication error in different care settings, such as in patients with several comorbidities and in surgical patients.

Medication reconciliation in surgical patients can reduce the occurrence of discrepancies requiring clarification, but its effect upon the prevention of reconciliation errors is not clear.

Medication reconciliation in cancer patients could improve the safety of medication use in these patients, although there are no randomized controlled trials to confirm these results.

\section{What this study adds}

Medication reconciliation increases drug safety in oncological patients, minimizing the reconciliation errors that reach the patient. More than one third of all patients would derive an improvement in safety from an exhaustive analysis of their current medication regimen at the start of a new chemotherapy regimen.

Medication reconciliation was shown to be valuable in this oncological patient population.

$\mathrm{M}$ edication reconciliation is defined as the formal and standardized process of obtaining the full list of drugs previously used by a patient, comparing it with the current drug regimen, and analyzing and resolving observed discrepancies. ${ }^{1,2}$ Medication reconciliation is a complex process that affects patients seen at different health care levels ${ }^{3}$ and is conducted with the ultimate aim of increasing the safety of medication use and reducing avoidable medication error.

Medication reconciliation programs may reduce reconciliation errors (REs) by 42\%-90\%. ${ }^{4}$ However, the true effectiveness of such programs remains to be established, since there is great heterogeneity in the reconciliation methodology used, which makes it difficult to compare the results of different studies. This heterogeneity mainly affects the types of REs recorded and the way in which home medications are recorded. ${ }^{4}$ Omission is the most common RE, although errors related to prescribed doses and regimens, nonjustified medications, duplicities, and interactions have also been described. ${ }^{5,6}$ Most reconciliation studies have been conducted in hospitalized patients, but little research has been carried out in oncological patients treated in an outpatient setting. ${ }^{7.8}$

Globally, cancer risk increases with age. As a result, its incidence can be expected to increase as the population life expectancy increases. ${ }^{9}$ Increased age is also associated with nononcological disease processes, such as arterial hypertension, diabetes, and chronic obstructive pulmonary disease, among other chronic disorders. In this regard, it is common for cancer patients receiving chemotherapy to also need outpatient treatment for these chronic disorders (e.g., blood pressure medications and antidiabetic agents). ${ }^{10,11}$ 


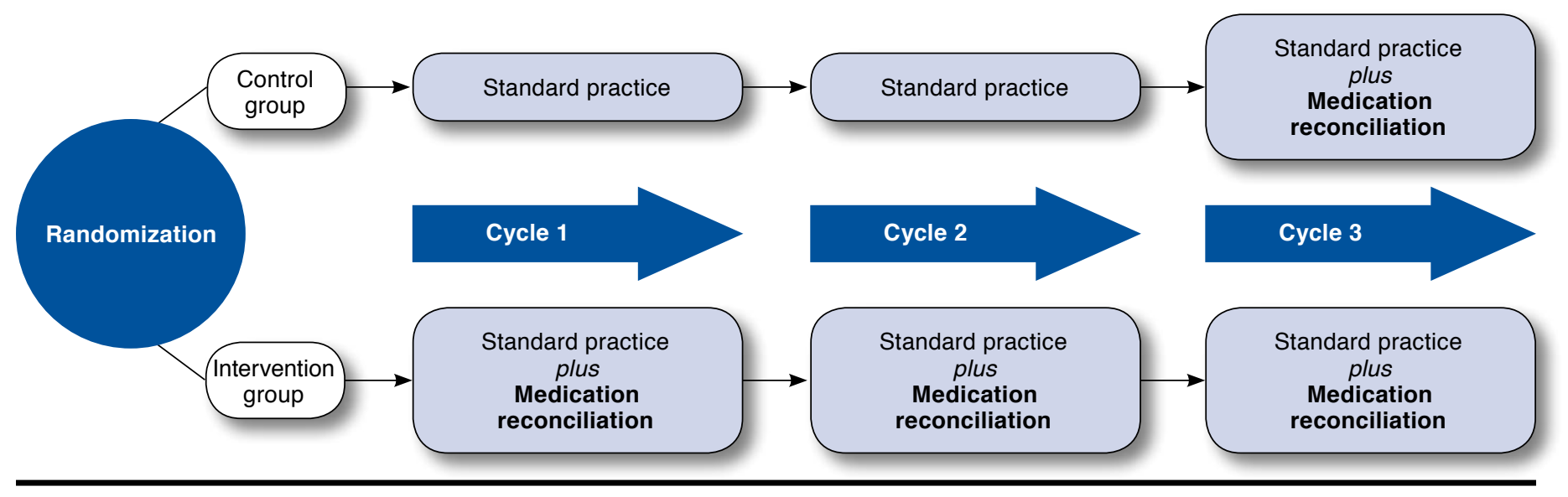

Patients receiving systemic cancer therapy are at high risk of drug interactions as a result of several concomitantly administered cancer drugs that include chemotherapy, hormone therapy, biological agents, and supportive therapies, in addition to medications prescribed as treatment for other coexisting disease states. ${ }^{12}$ At present, over $25 \%$ of all cancer treatments are administered orally, and some of these medications have a high potential risk of medication interactions with commonly used chronic medications. ${ }^{13}$ Approximately one third of all cancer patients receiving treatment as outpatients are exposed to possible interactions, although the incidence of true interactions is less clear. ${ }^{14}$

It would therefore be interesting to know whether the implementation of a medication reconciliation program reduces REs in these patients, potentially increasing the safety of drug treatment. The purpose of this study was to measure the effect of a medication reconciliation program on RE incidence in cancer patients receiving chemotherapy.

\section{Methods}

This clinical trial has been reported following the CONSORT checklist recommendations. ${ }^{15}$

\section{Trial Design}

A randomized, controlled, open-label clinical trial was designed. The study protocol was approved by the Clinical Research Ethics Committee of Puerta del Mar University Hospital and Distrito Bahía de Cádiz-La Janda (Spain).

\section{Participants}

This study was carried out in Puerta del Mar University Hospital (Cádiz, Spain), a tertiary care center with 620 beds. Patients over 18 years of age who started or changed chemotherapy in an outpatient setting for some oncological disorder and who were also receiving at least 1 additional outpatient medication on a chronic basis (prescription or over-the-counter medication) were included. The exclusion criteria included patients from whom a medication history could not be obtained due to cognitive impairment and/or the lack of a caregiver capable of supplying the required information.

\section{Intervention}

The patients in the intervention group entered a pharmacist-led medication reconciliation program that was specifically developed for cancer patients during the first cycle of chemotherapy (Figure 1). Standard practice for the control and intervention groups included validation of chemotherapy and supportive care medications in the treatment protocol: indication, dose, route and administration sequence, dose adjustments based on toxicity, and stability of intravenous preparations. Standard practice did not include medication reconciliation. The medication reconciliation program was applied to control patients in the third cycle of chemotherapy (Figure 1). Inclusion of the control patients in the third cycle allowed the analysis of REs in this group and thus establish differences with the intervention group.

The purpose of medication reconciliation in cancer patients is to reduce REs. Once all of a patient's medications have been reviewed, the existence or absence of discrepancies requiring clarification (DRCs) is determined. Reporting these DRCs to the physicians in charge of patient care, along with recommendations to resolve the discrepancies, is how REs were identified in this study.

Patients amenable to medication reconciliation were those who met the previously mentioned inclusion criteria. An electronic oncological prescription program (Oncowin) was used to identify patients receiving chemotherapy. This tool was used to obtain the following data: case history number, diagnosis, disease stage, age, gender, and chemotherapy and supportive med- 


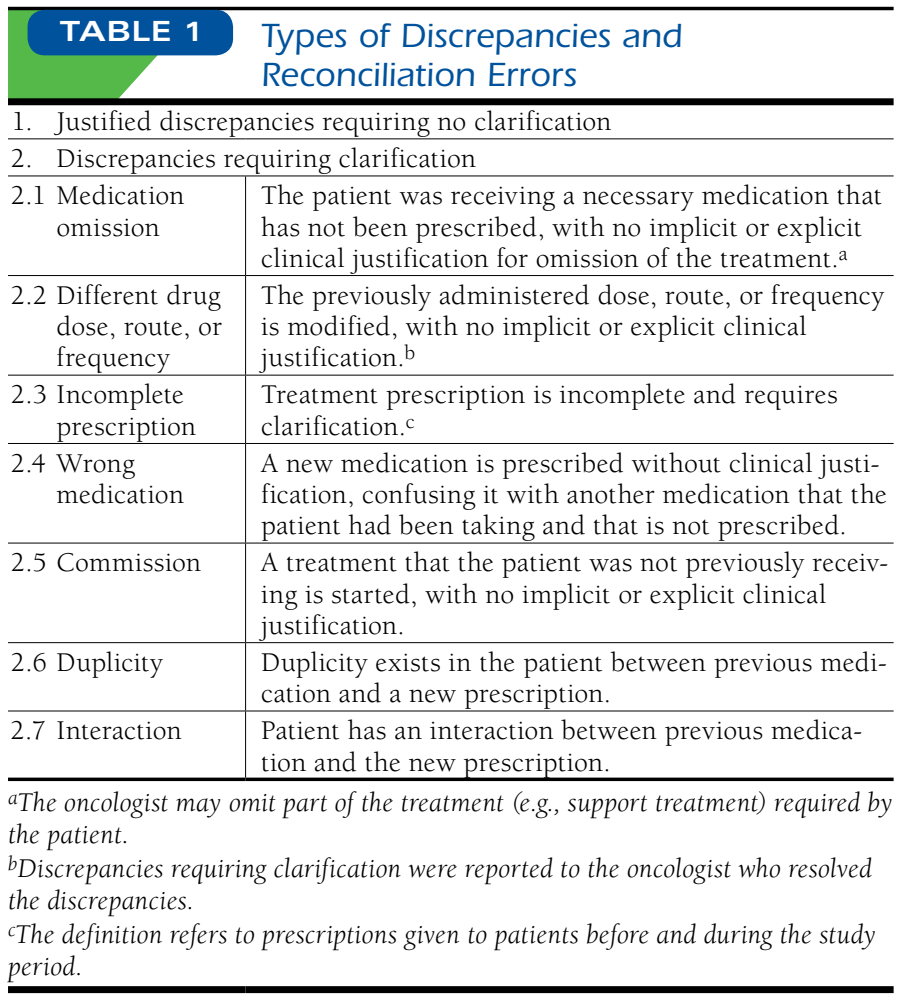

ications (drug, administration route, dosing, and frequency of administration). The treatment data contained in the electronic case history (Historia de Salud Única Digital-Diraya [HSUD]) were consulted for the information related to home treatment. HSUD identifies prescriptions that were filled for a patient and can capture pharmacy visitation data. In the patient interviews for this study, all drugs active in the HSUD in the last 90 days were considered. Performance status was recorded according to the Eastern Cooperative Oncology Group (ECOG) score and the Charlson Comorbidity Index (CCI) score. ${ }^{16}$

Patients were interviewed at their first visit to record their complete current treatments. This visit was made on day 1 of the first treatment cycle in the intervention group and on day 1 of the third cycle in the control group. On the second and subsequent visits, patient medication lists were checked first through the HSUD and then by patient questioning, in order to detect any changes in treatment. Following the interview, the detected REs were classified into types and subtypes (Table 1) according to the system proposed by Delgado-Sánchez et al. (2007). ${ }^{17}$

\section{Outcomes}

An RE was defined as any discrepancy reported to the physician in charge of patient care that resulted in a change in treatment in accordance with the clinical recommendation provided by the pharmacist. Discrepancies resolved by the pharmacist in 2 additional situations were also regarded as REs: (1) situations contemplated in the protocol jointly established with the Oncology Unit (need for dose adjustments in supportive care medication, e.g., antiemetic drugs) and (2) situations in which the drugs implicated in the DRCs were not related to medical prescription, since these drugs were overthe-counter products (including herbal remedies). Detected REs were classified into 2 groups: (1) those that were avoided and therefore did not reach the patient (RENRP) and (2) those that were detected after an RE had already occurred and thus reached the patient (RERP). Because RERP is a negative outcome from a patient safety standpoint, this error rate was established as the primary endpoint.

As secondary endpoints, the total number of REs, the number of REs per patient, the mean number of medications reconciled per patient, the total number of reconciled medications, the type of RE, and the drugs implicated in the RE (oncological vs. chronic medications) were recorded.

\section{Sample Size}

Calculation of the sample size was based on literature referring to the incidence of REs, with an incidence of nearly 30\% in surgical patients. ${ }^{18} \mathrm{~A}$ previous retrospective study of cancer patients in the Hospital Puerta del Mar found the percentage of individuals with DRCs to be $50.7 \%{ }^{19}$ According to the definition of RE given previously in the Outcomes section, 40\% of individuals could experience an RE. ${ }^{20}$ Using a reference incidence of RERP in the oncological setting of at least 30\%, it was estimated that the protocol intervention could reduce this incidence by half. ${ }^{18}$ Thus, assuming an alpha error of 5\%, a statistical power of $80 \%$, and considering a patient loss rate of $15 \%$, the calculated sample size was 142 patients.

\section{Randomization and Blinding}

Randomization (1:1) was carried out by random number assignment. The study information database was adapted for randomization purposes. Since the intervention was a professional act, blind patient assignment was not possible. The pharmacist compiled information about medications from the HSUD, as well as from primary care prescription records. This information was then verified by the pharmacist through a clinical patient interview. An open-label design was therefore adopted.

\section{Statistical Methods}

Statistical analysis was performed using the SPSS version 18 statistical package (SPSS, Chicago, IL). The differences in the primary endpoint between the intervention group and the control group were evaluated using the Fisher exact test. Descriptive statistics consisted of the mean and standard deviation (SD). 


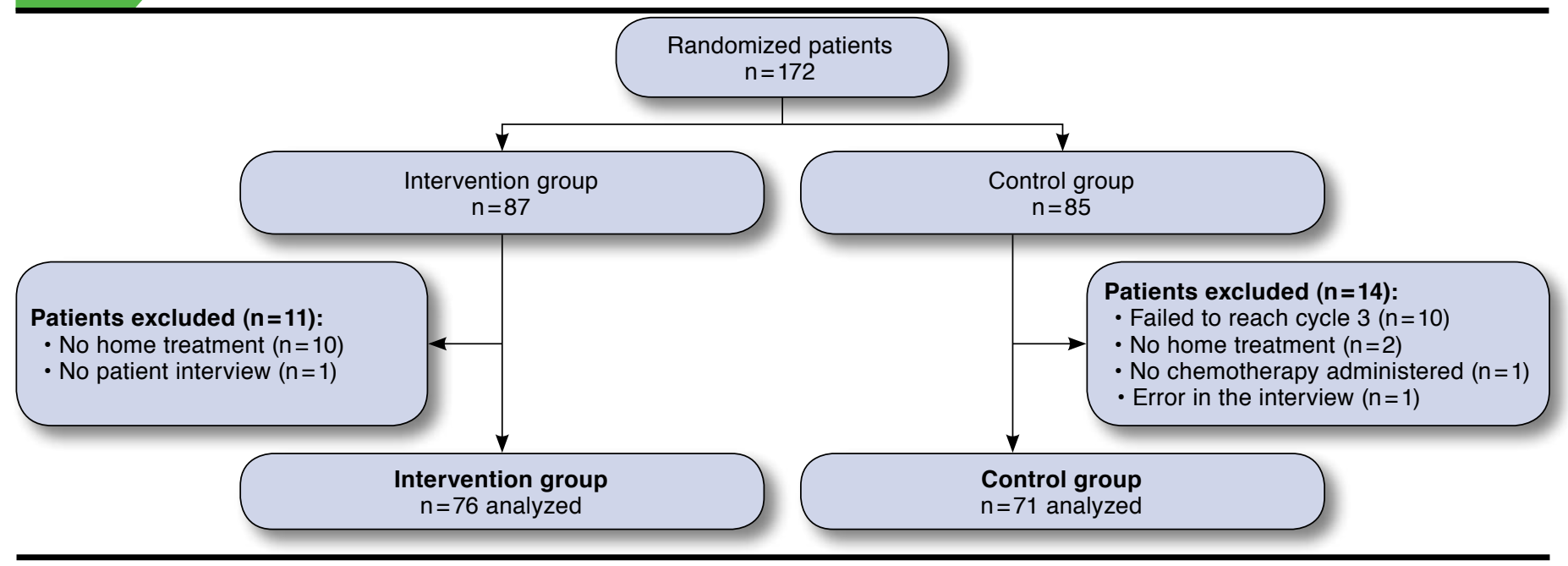

A prespecified analysis was made of the effects of the following variables on the incidence of REs independently of the study group: ECOG status, CCI score, and the degree of polymedication (number of medications used by patients). The criteria of Bjerrum et al. (1997) were used to establish the degree of poly-medication as follows: ${ }^{21}$ minor poly-medication $=2-3$ drugs, moderate poly-medication $=4-5$ drugs, and major polymedication $=$ over 5 drugs. The chi-squared test with Yates correction or, alternatively, the Fisher exact test, depending on the size of the comparator groups, was used to assess the relationship between the CCI score, ECOG score, and the degree of poly-medication with respect to RE occurrence. A value of $P<0.05$ was considered statistically significant.

\section{Results}

The clinical trial was carried out between February and September 2013, with the randomization of 172 patients, of which 147 were included (76 patients in the intervention group and 71 controls). Recruitment began in February 2013, with the last patient enrolled in May. The follow-up period ended in September 2013. The patient screening flowchart is shown in Figure 2. Patient characteristics were generally similar in both groups (Table 2). The distribution of some diagnoses differed between the 2 study arms (e.g., lung, stomach, and ovarian cancer).

Thirty-seven percent of the patients $(n=55)$ presented with at least $1 \mathrm{RE}$, totaling 87 (0.6 per patient). The number of patients with RERP (primary endpoint) totaled $3(4 \%)$ in the intervention group and 21 (30\%) in the control group (relative risk $[R R]=0.13,95 \%$ confidence interval $[C I]=0.04-0.43$; $P=0.0009$ ). The protocol intervention reduced RERP incidence by $26 \%$ (95\% CI $=14 \%-37 \% ; P<0.0001)$.
Independently of the patient group, a mean of 5.6 home medications were reconciled per patient (95\% CI $=5.1-6.0$ ), with 819 medications reconciled during the study period. In turn, RE distribution by type among the global patient series was as follows: interaction (94.3\%), duplicity (3.4\%), omission (1.1\%), and dose/frequency (1.1\%). The cancer medications most frequently implicated in REs are shown in Table 3.

The results of the prespecified analysis of the effects of the ECOG status, CCI score, and degree of poly-medication on the number of patients with REs are shown in Table 4. According to this analysis, the CCI score was not related to RE occurrence. However, the risk of RE was greater in patients with $E C O G \geq 2$ $(\mathrm{RR}=2.18,95 \% \mathrm{CI}=1.4-3.4 ; \mathrm{P}=0.018)$ and among patients with major poly-medication $(\mathrm{RR}=2.49,95 \% \mathrm{CI}=1.52-4.09 ; \mathrm{P}<0.001)$.

\section{Discussion}

This study demonstrates the need to implement pharmacistled medication reconciliation programs for cancer patients, since the proportion of patients with REs and the number of REs detected are at least equal to those found in other patient populations. ${ }^{22-27}$

In contrast to other studies, comparison with a control group allowed the measurement of the efficacy of medication reconciliation for avoiding RERP, which was found to be statistically significant $(R R=0.13,95 \% C I=0.04-0.43)$. Since intervention during the third chemotherapy cycle proved necessary in order to determine RE incidence in the control group, the sustained effect of the intervention over time is not known. Nevertheless, the results obtained with intervention in the first treatment cycle in the intervention group were found to be sufficiently favorable to allow the recommendation of such practice in cancer patients. 


\begin{tabular}{|c|c|c|c|c|}
\hline \multirow[b]{2}{*}{ Gender } & \multicolumn{2}{|c|}{ Intervention } & \multicolumn{2}{|c|}{ Control } \\
\hline & $\mathrm{N}$ & $\%$ & $\mathrm{~N}$ & $\%$ \\
\hline Male & 37 & 49 & 28 & 39 \\
\hline Female & 39 & 51 & 43 & 61 \\
\hline \multirow[t]{2}{*}{ Age } & Mean & SD & Mean & SD \\
\hline & 60.2 & 13.2 & 60.7 & 12.4 \\
\hline Diagnosis & $\mathrm{N}$ & $\%$ & $\mathrm{~N}$ & $\%$ \\
\hline Colorectal & 20 & 26 & 21 & 29 \\
\hline Lung & 17 & 22 & 7 & 10 \\
\hline Breast & 16 & 21 & 13 & 18 \\
\hline Head and neck & 5 & 6 & 5 & 7 \\
\hline Stomach & 4 & 5 & - & - \\
\hline Esophagus & 2 & 3 & 3 & 4 \\
\hline Cervix & 2 & 3 & - & - \\
\hline Pancreas & 2 & 3 & 3 & 4 \\
\hline Sarcoma & 2 & 3 & - & - \\
\hline Others & 7 & 9 & 10 & 14 \\
\hline Ovary & - & - & 8 & 11 \\
\hline Bladder & - & - & 2 & 3 \\
\hline ECOG status & $\mathrm{N}$ & $\%$ & $\mathrm{~N}$ & $\%$ \\
\hline 0 & 35 & 46 & 30 & 42 \\
\hline 1 & 26 & 34 & 23 & 32 \\
\hline 2 & 3 & 4 & 6 & 8 \\
\hline 3 & 2 & 3 & 0 & 0 \\
\hline Not known & 10 & 13 & 12 & 17 \\
\hline \multirow[t]{2}{*}{ CCI score } & Mean & SD & Mean & SD \\
\hline & 5.1 & 2.2 & 5.4 & 2.3 \\
\hline
\end{tabular}

Patient characteristics were generally similar in both groups (intervention and control). However, there were differing diagnoses between them (e.g., lung, stomach, and ovarian cancer), as well as a different gender distribution, with more women than men in the control group (61\% vs. $51 \%$ ). No information was available on whether these differences may have affected the incidence of REs, although there are data on how these differences can influence the appearance of DRCs. In a retrospective study of colon and breast cancer patients, gender and diagnosis had no effect on the incidence of DRCs. ${ }^{7}$ It can be assumed that these parameters also have no effect upon RE incidence, with no influence upon the primary study endpoint in this study.

Most of the patients included in this study (77.5\%) had an ECOG score of 0-1 and were therefore in good general health. Regarding the relationship between the ECOG score and RE occurrence, an association was found between the risk of RE and $E C O G \geq 2$. This finding did not depend on the number of medications, which was not different between patients with

\begin{tabular}{|c|c|c|c|}
\hline & \multirow[b]{2}{*}{ RE (\%) } & \multicolumn{2}{|c|}{ Type of RE } \\
\hline & & Duplicity (\%) & Interactions (\%) \\
\hline \multicolumn{4}{|l|}{ Cancer treatment } \\
\hline Dexamethasone & 48 & 2.3 & 44.7 \\
\hline Ondansetron & 8 & & 8 \\
\hline Erlotinib & 6 & & 6 \\
\hline Vinorelbine & 4 & & 4 \\
\hline Capecitabine & 4 & & 4 \\
\hline Cyclophosphamide & 4 & & 4 \\
\hline Cisplatin & 4 & & 4 \\
\hline \multicolumn{4}{|c|}{ Chronic outpatient medications $^{a}$} \\
\hline Metformin & 15 & & 15 \\
\hline Insulin & 8 & & 8 \\
\hline Omeprazole & 6 & & 6 \\
\hline Ibuprofen & 6 & & 6 \\
\hline Dexamethasone & 3 & & 3 \\
\hline Acetylsalicylic acid & 3 & & 3 \\
\hline Echinacea & 3 & & 3 \\
\hline Hydrochlorothiazide & 3 & & 3 \\
\hline
\end{tabular}

ECOG scores of $0-1$ and those with ECOG $\geq 2$ (5.5 vs. 6.4 medications per patient; $P=0.326$ ). The strength of this comparison is limited by the few patients that presented an ECOG score of $\geq 2$. A larger study population would be needed, along with a multivariate analysis, in order to determine which variables are associated with an increased risk of RE.

The CCI comprises 19 items related to comorbidities that, when present, have been shown to exert a concrete influence upon patient life expectancy. This index has been used in other studies of cancer patients as a predictor of overall survival. ${ }^{11,28,30}$ The CCI score was balanced in both study arms and was high (a score of over 5 is associated with an 85\% mortality rate after 3 years), reflecting a poor prognosis among these patients. No relationship was found between the CCI score and RE incidence.

Likewise, there were no differences between the 2 groups in the number of medications received by each patient $(P>0.05)$. However, the number of patients with major poly-medication according to the criteria of Bjerrum et al. was found to be greater in the intervention group. ${ }^{21}$ This may result in a higher RE incidence in the intervention group. Consequently, detection of REs could be related not only to the intervention itself but also to the presence of a higher incidence of REs in these patients.

An important difference between this study and other publications found in the literature refers to the type of RE recorded. According to the literature, the main type of RE is the omission of home medication. ${ }^{20,22,25-27,31,32}$ In this study, only 1 case of a major RE due to omission was recorded. In contrast, $94.3 \%$ of 
TABLE 4 Prespecified Analysis of Effects of ECOG Status, CCI Score, and Degree of Poly-Medication on Number of Patients with REs

\begin{tabular}{|c|c|c|c|c|c|c|}
\hline & $\mathrm{N}$ & Total & $\%$ & $\mathrm{RR}$ & \multicolumn{2}{|c|}{$95 \% \mathrm{CI}$} \\
\hline \multicolumn{7}{|l|}{ ECOG status } \\
\hline $0-1$ & 38 & 114 & 33 & & & \\
\hline$\geq 2$ & 8 & 11 & 73 & 2.18 & 1.4 & 3.41 \\
\hline \multicolumn{7}{|l|}{ CCI score } \\
\hline$<5$ & 22 & 51 & 43 & & & \\
\hline$\geq 5$ & 33 & 96 & 34 & 0.80 & 0.52 & 1.21 \\
\hline \multicolumn{7}{|l|}{ Poly-medication $^{a}$} \\
\hline Minor-Moderate & 15 & 71 & 21 & & & \\
\hline Major (>5 drugs) & 40 & 76 & 53 & 2.49 & 1.52 & 4.09 \\
\hline
\end{tabular}

all REs were attributable to interactions of different severities, compared with only 5\% in another study. ${ }^{18}$ Such discrepancies could be explained mainly by differences in the health care setting involved. The cancer patients included in this study received chemotherapy in an outpatient setting. In this setting, the oncologist only prescribes chemotherapeutic and supportive treatment, without having to consider chronic therapies. The risk of omission of home medication is therefore minimized, since in most cases, the oncologist does not deal with this type of treatment.

\section{Limitations}

As previously mentioned in the Discussion section, there were differing diagnoses between the intervention and control groups, as well as a different gender distribution, with more women than men in the control group ( $61 \%$ vs. $51 \%$ ). No information was available on whether these differences affected the incidence of REs.

The number of patient losses was higher in the control group than in the intervention group. This result happened because controls who failed to reach the third treatment cycle were not interviewed and were consequently excluded. The fact that patients might not reach the third chemotherapy cycle because of the seriousness of their conditions-who then may not be available for the reconciliation interview-was not taken into account. This did not happen in the intervention group, which may constitute a limitation of the study design.

\section{Conclusions}

We have compared the effect of medication reconciliation versus standard practice in cancer patients receiving chemotherapy and have observed a notable decrease in RERP incidence. The factors that may influence RE occurrence in oncological patients have not been fully established, although parameters such as the degree of poly-medication and performance status may play a role.

\section{Authors}

TRIANA GONZÁLEZ-CARRASCOSA VEGA, Pharmacist, Area de Gestión Sanitaria Norte de Cádiz, Spain, and JESÚS FRANCISCO SIERRA SÁNCHEZ, PharmD, Department of Pharmacy, Area de Gestión Sanitaria Norte de Cádiz, Spain. MARÍA JOSÉ MARTÍNEZ BAUTISTA, BCOP Pharmacist; FÁTIMA GARCÍA-MARTÍN, Pharmacist; and FRANCISCO SUÁREZ-CARRASCOSA, Pharmacist, Department of Pharmacy, Hospital Universitario Puerta del Mar, Cádiz, Spain. JOSE MANUEL BAENA-CANADA, PhD, Department of Oncology, Hospital Universitario Puerta del Mar, Cádiz, Spain.

AUTHOR CORRESPONDENCE: Triana González-Carrascosa Vega, Hospital de Jerez, Ronda de Circunvalación s/n, 11407, Jerez de la Frontera, Cádiz, Spain. Tel.: +34670943647;

E-mail: trianaglez-carrascosavega@hotmail.com.

\section{DISCLOSURES}

No outside funding supported this study. The authors declare that they have no affiliations with or financial interests in any company, product, or service described in the manuscript.

Study concept and design were contributed by Sierra-Sánchez, MartínezBautista, Baena-Cañada, and González-Carrascosa Vega. Martínez-Bautista, García-Martín, Suárez-Carrascosa, and González-Carrascosa Vega collected the data, which was interpreted by Sierra-Sánchez, Martínez-Bautista, BaenaCañada, and González-Carrascosa Vega. The manuscript was written by Sierra-Sánchez and González-Carrascosa Vega and revised by Sierra-Sánchez, Martínez-Bautista, Baena-Cañada, and González-Carrascosa Vega.

\section{REFERENCES}

1. Joint Commission Accreditation Healthcare Organizations. Comprehensive Accreditation Manual for Hospitals (CAMH): The Official Handbook. Oakbrook Terrace, IL: Joint Commission Resources, 2006.

2. Institute for Healthcare Improvement. How-to guide: prevent adverse drug events (medication reconciliation). Revised March 2016. Available at: http://www.ihi.org/resources/Pages/Tools/HowtoGuidePrevent AdverseDrugEvents.aspx. Accessed April 3, 2016. 
3. Gleason KM, Brake H, Agramonte V, Perfetti C. Medications at transitions and clinical handoffs (MATCH) toolkit for medication reconciliation. (Prepared by the Island Peer Review Organization, Inc., under Contract No. HHSA2902009000 13C). AHRQ Publication No. 11(12)-0059. Rockville, MD: Agency for Healthcare Research and Quality. Revised August 2012. Available at: http://www.ahrq.gov/sites/default/files/publications/files/match. pdf. Accessed March 18, 2016.

4. Alfaro-Lara ER, Vega-Coca MD, Galván-Banqueri M, et al. [Pharmacological treatment conciliation methodology in patients with multiple conditions]. Aten Primaria. 2014;46(2):89-99. [Article in Spanish]

5. Gleason KM, McDaniel MR, Feinglass J, et al. Results of the Medications at Transitions and Clinical Handoffs (MATCH) study: an analysis of medication reconciliation errors and risk factors at hospital admission. J Gen Intern Med. 2010;25(5):441-47.

6. Conklin JR, Togami JC, Burnett A, et al. Care transitions service: a pharmacy-driven program for medication reconciliation through the continuum of care. Am J Health Syst Pharm. 2014;71(10):802-10.

7. González-Carrascosa Vega T, Sierra-Sánchez JF, Martínez-Bautista MJ, et al. Discrepancies requiring clarification in cancer patients: a risk predictive model. Eur J Hosp Pharm. 2013;20(2):93-96.

8. Salamin LB, Eschenburg K, Kraft S, Mackler E. Evaluation of outpatient medication reconciliation involving student pharmacists at a comprehensive cancer center. J Am Pharm Assoc (2003). 2015;55(5):540-45.

9. Coleman MP. Cancer survival: global surveillance will stimulate health policy and improve equity. Lancet. 2014;383(9916):564-73.

10. Nicholas Z, Hu N, Ying J, Soisson P, Dodson M, Gaffney DK. Impact of comorbid conditions on survival in endometrial cancer. Am J Clin Oncol. 2014;37(2):131-34.

11. Bøje CR, Dalton SO, Primdahl H, et al. Evaluation of comorbidity in 9388 head and neck cancer patients: a national cohort study from the DAHANCA database. Radiother Oncol. 2014;110(1):91-97.

12. van Leeuwen RW, Brundel DH, Neef C, et al. Prevalence of potential drug-drug interactions in cancer patients treated with oral anticancer drug. Br J Cancer. 2013;108(5):1071-78.

13. Conde-Estevez D, Salas E, Albanell J. Survey of oral chemotherapy safety and adherence practices of hospitals in Spain. Int J Clin Pharm. 2013;35(6):1236-44.

14. Riechelmann RP, Del Giglio A. Drug interactions in oncology: how common are they? Ann Oncol. 2009;20(12):1907-12.

15. Moher D, Hopewell S, Schulz KF, et al. CONSORT 2010 explanation and elaboration: updated guidelines for reporting parallel group randomised trials. BMJ. 2010;340:c869.

16. Charlson M, Pompei P, Ales KL, et al. A new method of classifying prognostic comorbidity in longitudinal studies: development and validation. J Chron Dis. 1987;40(5):373-83.

17. Delgado Sánchez O, Anoz Jiménez L, Serrano Fabiác A, Nicolás Picó J. [Conciliation in medication]. Med Clin (Barc). 2007;129(9):343-48. [Article in Spanish]
18. Kwan Y, Fernandes OA, Nagge JJ, et al. Pharmacist medication assessments in a surgical preadmission clinic. Arch Intern Med. 2007;167(10):1034-40.

19. González-Carrascosa Vega T, Sierra Sánchez JF, Martínez Bautista MJ, et al. Identificación de factores de riesgo en la conciliación farmacológica del paciente oncológico. Aten Farm. 2012;14(4):281-84.

20. Delgado Sánchez O, Nicolás Picó J, Martínez López I, Serrano Fabiá A, Anoz Jiménez L, Fernández Cortés F. [Reconciliation errors at admission and departure in old and polymedicated patients. Prospective, multicenter randomized study]. Med Clin (Barc). 2009;133(19):741-44. [Article in Spanish]

21. Bjerrum L, Rosholm JU, Hallas J, Kragstrup J. Methods for estimating the occurrence of polypharmacy by means of a prescription database. Eur J Clin Pharmacol. 1997;53(1):7-11.

22. Salanitro AH, Osborn CY, Schnipper JL, et al. Effect of patient and medication-related factors on inpatient medication reconciliation errors. J Gen Intern Med. 2012;27(8):924-32.

23. Knez L, Suskovic S, Rezonja R, Laaksonen R, Mrhar A. The need for medication reconciliation: a cross-sectional observational study in adult patients. Respir Med. 2011;105(Suppl 1):S60-66.

24. Gizzi LA, Slain D, Hare JT, Sager R, Briggs F 3rd, Palmer CH. Assessment of a safety enhancement to the hospital medication reconciliation process for elderly patients. Am J Geriatr Pharmacother. 2010;8(2):127-35.

25. Unroe KT, Pfeiffenberger T, Riegelhaupt S, Jastrzembski J, Lokhnygina Y, Colón-Emeric C. Inpatient medication reconciliation at admission and discharge: a retrospective cohort study of age and other risk factors for medication discrepancies. Am J Geriatr Pharmacother. 2010;8(2):115-26.

26. Pippins JR, Gandhi TK, Hamann C, et al. Classifying and predicting errors of inpatient medication reconciliation. J Gen Intern Med. 2008; 23(9):1414-22

27. Nickerson A, MacKinnon N, Roberts N, Saulnier L. Drug-therapy problems, inconsistencies and omissions identified during a medication reconciliation and seamless care service. Health Q. 2005;8(Spec No):65-72.

28. Robbins JR, Gayar OH, Zaki M, Mahan M, Buekers T, Elshaikh MA. Impact of age-adjusted Charlson comorbidity score on outcomes for patients with early-stage endometrial cancer. Gynecol Oncol. 2013;131(3):593-97.

29. Briganti A, Spahn M, Joniau S, et al. Impact of age and comorbidities on long-term survival of patients with high-risk prostate cancer treated with radical prostatectomy: a multi-institutional competing-risks analysis. Eur Urol. 2013;63(4):693-701.

30. Simon TG, Beland MD, Machan JT, Dipetrillo T, Dupuy DE. Charlson Comorbidity Index predicts patient outcome, in cases of inoperable nonsmall cell lung cancer treated with radio frequency ablation. Eur J Radiol. 2012;81(12):4167-72.

31. Ledger S, Choma G. Medication reconciliation in hemodialysis patients. CANNT J. 2008;18(4):41-43.

32. Zoni AC, Durán García ME, Jiménez Muñoz AB, Salomón Pérez R, Martin P, Herranz Alonso A. The impact of medication reconciliation program at admission in an internal medicine department. Eur J Intern Med. 2012;23(8):696-700. 